\title{
Hailey-Hailey Disease (Benign Familial Pemphigus): Carbon Dioxide Laser Therapy
}

\author{
Anatoli Freiman, BSc ${ }^{* \dagger}$, Miriam D. Hakim MD ${ }^{\dagger}$, Robin C. Billick, MD ${ }^{\dagger}$.
}

\section{INTRODUCTION}

Familial benign chronic pemphigus (Hailey-Hailey disease) is a rare autosomal-dominant genodermatosis, characterized by recurrent skin eruptions mostly in the intertriginous areas. The clinical manifestations consist of closely grouped small vesicles with predilection for neck, axillae and groin areas. The vesicles usually progress to vegetating fissured plaques with bullae and erosions. Familial benign pemphigus differs from other forms of pemphigus in its genetic pattern, as well as by itsabsence of mouth lesions and absence of intercellular antibodies (1). Hailey-Hailey disease can be a chronic, debilitating condition, both physically and psychologically. Despite a wide variety of topical and systemic medical treatments, this illness presents a major therapeutic challenge due to a high recurrence rate. Review of the recent literature demonstrates that surgical modalities may offer the benefit of clearing active lesions with possible eradication of the disease in the treated areas. We present the successful management of a patient with Hailey-Hailey disease, unresponsive to conventional treatment, with a short pulse carbon dioxide laser therapy.

\section{CASE REPORT}

Our patient is a 34 y.o. white male with a longstanding history of vesicles, bullae and erosions in both axillae. The lesions would appear in crops and last for several weeks, aggravating during the summer months. His symptoms of pruritus, burning, pain, and malodor in the axillae caused significant discomfort, and made his occupation as a cleaner more difficult.

*To whom correspondence should be addressed.

$\nmid$ Division of Dermatology, SMBD-Jewish General Hospital, SMBDJewish General Hospital, 3755 Côte-Ste-Catherine, G-026, Montreal, QC, H3T 1E2, Canada. E-Mail: freim02@med.mcgill.ca
The patient's family history was remarkable for his mother having similar eruptions.

Physical examination in our dermatology clinic revealed a young man in mild distress. Involving the right and left axillae, he had dry, crusted, scaling, rustcolor plaques and erosions, with erythema more pronounced at the borders (Fig 1). The nape of the neck and the groin area were also mildly involved. The rest of cutaneous examination was unremarkable.

A 4-0mm punch biopsy specimen was taken from the affected axillar tissue. It demonstrated epidermal hyperplasia with presence of multifocal suprabasilar and intraepidermal acantholytic vesicles. The acantholytic cells had a "dilapidated brick wall" appearance. Histhopathologic diagnosis was consistent with Hailey-Hailey disease, otherwise known as benign familial pemphigus.

Prior to our treatment with the $\mathrm{CO}_{2}$ laser, the patient had received conventional therapy, including topical antibiotics, antifungals and high potency steroids. Despite the best medical management, the patient continued experiencing major exacerbations without significant time duration between disease flares. Because of the refractory disease, the patient decided to pursue $\mathrm{CO}_{2}$ laser therapy of his axillae, which were the main sites of discomfort. We went on to utilize the UltraPulse 5000 carbon dioxide laser and treated both axillae with four passes $(300 \mathrm{~mJ}, 60$ watts, 200 pulses/sec settings). The surgery was successful, and there was a dramatic clearing of the active lesions immediately following the procedure (Fig 2). Postoperatively, the patient applied petroleum jelly to the surgical site until the wounds healed. Complete reepithelization occurred within two weeks, and there has been no recurrence of the lesions noted in either axillar area during the 12-month follow-up period, which is still ongoing. 


\section{DISCUSSION}

Hailey-Hailey disease (Benign familial pemphigus) was first described 1939 by the Hailey brothers (2). This rare blistering disease is an autosomal dominantinherited genodermatosis with incomplete penetrance. A positive family history is present in approximately two thirds of patients (3), while the rest of cases are believed to be new mutations, involving a defect in a calcium ATPase.

The pathophysiology of Hailey-Hailey disease is still not fully understood. Reports in the literature note that the underlying pathologic process is acantholysis and that the fragility of epidermis is secondary to a defect in the adhesion complex between desmosomal proteins and tonofilaments (4). This defect appears to involve interfollicular epidermal cells, whereas the adnexal keratinocytes are usually spared of the acantholytic process $(5,6)$. Histologically, a widespread incomplete suprabasal acantholysis is the trademark of HaileyHailey disease, causing the well-known "dilapidated brick wall" appearance of the lower epidermis (5).

The clinical findings of Hailey-Hailey disease include vesicles and bullae arising on apparently normal skin. After the bullae rupture, erosions are seen, which may impetiginize. The condition manifests predominantly in the axillae, groin and intertriginous areas. The marked predilection for these sites is believed to occur because of the dense population by adnexal structures such as hair follicles, apocrine and eccrine glands. HaileyHailey usually appears only after puberty, as the adnexae mature (7). Clinically, the differential diagnosis includes intertrigo, candidiasis, and frictional or contact dermatitis (4)

The onset is mostly between the second and third decade of life and is triggered by friction and excessive sweating (6). It has been postulated that benign familial pemphigus may be expressed in any part of the body when various external stimuli insult the skin with an underlying primary defect (3). These triggers include trauma, friction, warm and humid environment, UV radiation, contact allergens (including ingredients in topical therapy), and infectious agents (bacteria, yeasts, HSV). A case of total-body generalized Hailey-Hailey disease has been reported in the literature (8). Superinfection of the lesions, particularly by Staphylococcus aureus or Candida species, is common, and is a trigger for further acantholysis and maintenance of pathologic process.

Hailey-Hailey has a variable, usually chronic course, with periods of remissions and exacerbations. Recurrences are more often and more severe in the hot summer months. The condition is often debilitating, both physically and psychologically. The patients suffer

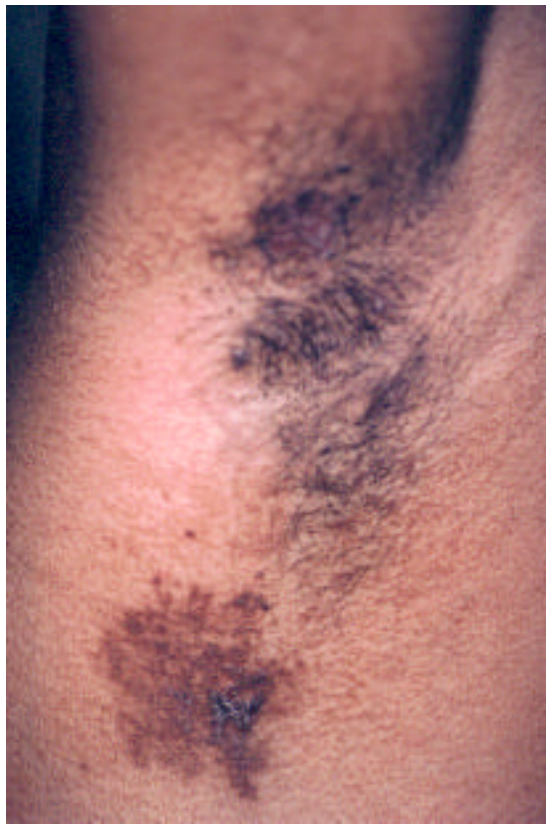

Figure 1: Pre-operative photograph of dry crusted plaques in the patient's left axillar area.

from pruritus, burning, intense pain and restricted mobility, which can be depressing. Malodorous discharge greatly affects social activity and patient's lifestyle. In severe cases, the condition may give rise to temporary or permanent disability.

The treatment of Hailey-Hailey poses a challenge. Numerous conservative modalities have been used, ranging from topical and systemic therapy with antibiotics, antifungals, and coticosteroids to dapsone, methotrexate, thalidomide, etretinate and even cyclosporine $(3,5,9)$. Whereas these treatment approaches may improve or even control the disease in a short term, they have not been shown to be effective on a long-term basis or for severe chronic forms of relapsing disease.

Surgical intervention has been introduced to control difficult cases refractive to medical therapy. The first successful report came in 1966 from Biro and Madday, who performed full-thickness excision of lesional skin followed by split-thickness grafting from the thigh (10). Kumar described the case of a patient with HaileyHailey disease who was treated by excision of lesional skin and subsequent primary closure of the wound (3). Since then, several authors presented favorable results obtained by excision of involved skin with subsequent grafting onto lesional sites. However, because of the morbidity and potential complications associated with wide excision of intertriginous folds (scar contracture with subsequent restricted mobility, graft failure, infection, thromboembolic disease and poor cosmetic results (9), alternative modalities had to be pursued. 


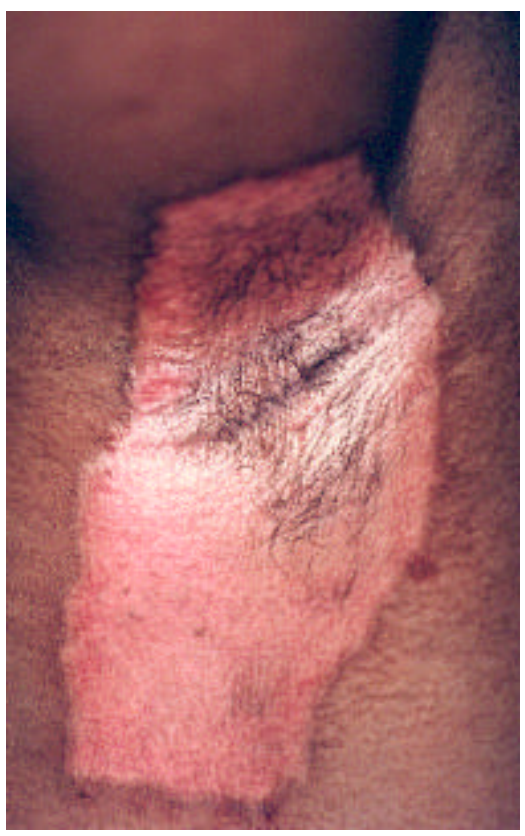

Fig 2: Two-week post-operative photograph of the patient's axillar area. Remarkable clearing of lesions is seen.

Belhaouari et al. (11) first suggested dermabrasion therapy in 1983, and in 1989 Hamm described successful dermabrasion in four cases of the HaileyHailey disease resistant to conventional management (12). Kirtschig et al. (12) subsequently reported two cases of patients in whom dermabrasion led to a longstanding absence of active skin lesions. Metze et al. (13) performed histological, ultrastractural and histochemical study of lesional and non-lesional skin of 18 patients with Hailey-Hailey disease. They note that none of the adnexal epithelia expressed the intrinsic defect of cell adhesion. Their finding offered an explanation for the success of dermabrasion - after complete removal of the involved epidermis, reepithelization would occur from the skin appendages.

Recently, the use of carbon dioxide laser emerged as an effective option for treatment of numerous epidermal and dermal dermatological lesions, including benign and malignant growths, keloids, vascular deformities, warts, tattoos, etc. (14). The first report of successful carbon dioxide laser abrasion of Hailey-Hailey disease came in 1987 from Don et al. (3), who treated the inner aspect of the left thigh of a 50-year old man. The area remained disease-free during an 8-month follow-up period, as contrasted to the untreated opposite thigh. Kartamaa and Reitamo (15) described the use of continuous CO2 laser in six patients with Hailey-Hailey disease, demonstrating substantial improvement of disease-affected areas in most patients post $\mathrm{CO}_{2}$ laser. The therapeutic success of the laser is attributed to its ability to selectively destroy the diseased epidermis while leaving the dermal structures intact (14). The adnexae, which presumably do not express the adhesion defect are responsible for re-epithelization following the ablation of the affected keratinocytes (6). Compared to dermabrasion, $\mathrm{CO} 2$ laser ablation is a careful, low bleeding method with less postoperative pain and rapid healing of the erosions. It also offers a safer work environment relative to dermabrasion.

More recently, Christian and Moy (9) suggested the use short-pulsed short-dwell carbon dioxide laser for the treatment of Hailey-Hailey disease. Lasers with short pulse durations generally cause less residual thermal damage than the ones with relatively long pulse duration, and are therefore associated with less erythema and faster healing. The authors presented a case of a 26-year-old woman with a 10-year history of refractory axillary Hailey-Hailey disease. They treated the right axilla with two passes, and the left one with three passes, repeating the procedure, with two additional passes, three years later. At a 3.5-year followup, recurrence was noted in the right axilla.

We went on to use UltraPulse 5000 short pulse laser therapy at four passes on our patient. The benefit of our intervention is demonstrated by the absence of recurrence of active lesions in either axilla over a 12month follow-up period, which is still ongoing. We suggest that short pulse $\mathrm{CO}_{2}$ laser therapy is a valuable treatment modality of chronic, symptomatic, localized plaques of benign familial pemphigus, particularly when conservative therapy has been unsuccessful. Further controlled studies, with more patients and longer follow-up periods will be needed to determine optimal laser settings, as well as to establish the longterm effects of the therapy and duration of remission.

\section{REFERENCES}

1. Goldman L, Bennett JC: Cecil Textbook of Medicine 21st ed. p 2284. W. B. Saunders 2000.

2. Hailey H, Hailey H. Familial benign chronic pemphigus. Arch Dermatol 1939; 39: 679-685.

3. Don PC, Carney PS, Lynch WS, et al. Carbon Dioxide Laserabrasion: A new approach to management of familial benign chronic pemphigus (Hailey-Hailey Disease). J Dermatol Surg Oncol 1987 Nov; 13: 1187-94.

4. Fitzpatrick TB, Johnson RA, Wolff K, Suurmond D. Color Atlas and Synopsis of Clinical Dermatology 4th ed. McGraw Hill 2001.

5. Hamm H, Matze D, Brocker EB. Hailey-Hailey Disease. Eradication by Dermabrasion. Arch Dermatol 1994 Sept; 130: 1143-1149.

6. Touma DJ, Krauss M, Feingold DS. Benign familial pemphigus. Treatment with the pulsed carbon-dioxide laser. Dermatol Surg 1998 Dec; 24(12): 1411-4.

7. Michel B. Commentary: Hailey-Hailey Disease. Arch Dermatol 1982; 118: 781-3.

8. Marsch WC, Stuggen G. Generalized Hailey-Hailey disease. Br J Dermatol 1978; 99: 553-560. 
9. Christian MM, Moy RL. Treatment of Hailey-Hailey disease using short pulsed and short dwell time carbon dioxide lasers. Dermatol Surg 1999 Aug, 25(8): 661-3.

10. Biro FA, Maday P. Familial chronic pemphigus. Arch Dermatol 1969; 100: 385.

11. Belhaouari L, Chavignon J, Cantala $\mathrm{P}$ et al. Indications peu communes de dermabrasion. Ann Dermatol Venerol 1983; 110: 298.

12. Kirtschig G, Gieler U, Happle R. Treatment of Hailey-Hailey disease by dermabrasion. J Am Acad Dermatol 1993; 28: 784
6.

13. Metze D, Hamm H, Schorat A, Luger T. Involvement of the adherens junction-actin filament system in acantholytic dyskeratosis of Hailey-Hailey disease. J Cutan Pathol 1996 Jun; 23(3): 211-22.

14. Hruza G. Lasers in Dermatology. Laser treatment of Warts and Other Epidermal and Dermal Lesions. Dermatol Clinics 1997 July; 15(3): 487-505

15. Kartamaa M, Reitamo S. Familial Benign Chronic Pemphigus (Hailey-Hailey Disease). Arch Dermatol 1992 May; 646-8.

Anatoli Freiman is currently a fourth year medical student at McGill University. He holds a B.Sc. degree in Physiology from McGill (Montreal, Quebec, Canada). His aspiration is to pursue a career in academic dermatology. Miriam Hakim, M.D. is in her fourth year of dermatology residency training at McGill University (Montreal, Quebec, Canada). She holds an MD degree from the University of Cincinnati School of Medicine and a BSIE degree from University of Cincinnati College of Engineering. Robin C. Billick, M.D., MSc., FRCPC received his MD from McGill University (Montreal, Quebec, Canada). He subsequently completed his residency training in internal medicine at McGill and dermatology training at the University of Miami, Florida. Dr. Billick is presently Chief of Dermatology at the SMBD-Jewish General Hospital in Montreal, Quebec, Canada. 